\title{
The dynamics of questions and focus
}

\author{
Maria Aloni and Robert van Rooy \\ ILLC/University of Amsterdam
}

\section{Background and Motivations}

This article presents a dynamic account of questions and focus which combines the logical appeal of the partition theory of questions with the empirical strength of the structured meaning account of questions and focus.

A dynamic analysis which treats meanings as context change potentials (e.g. Stalnaker, Kamp, Heim, Groenendijk \& Stokhof) will provide us with a substantial account of the dependence of focused answers on the context set up by their preceding questions. Questions pose conditions on the focal structure of their answers (Paul 1880) and can further restrict the domain of subsequent focusing operators like only (e.g. Zimmermann, von Stechow 1991, Jäger 1996, von Fintel 1995). As an illustration consider the following example:

(1) a. Who did John introduce to Sue?

b. Which gentlemen did John introduce to Sue?

c. John introduced only $[\text { Bill }]_{F}$ to Sue.

d. *John introduced only Bill to $[\text { Sue }]_{F}$.

After question (1a) or (1b), only an answer with the focal structure in (1c) is felicitous or congruent. Answer (1d) is out. Consider now the congruent answer in (1c). After question (1a), answer (1c) means 'The only person John introduced to Sue is Bill'. After (1b), it can mean 'The only gentleman John introduced to Sue is Bill'.

Standard analyses of focus define congruence in terms of identity between the question meaning and the focal alternatives of the answer (e.g. von Stechow 1991, Roberts 1996), and identify the domain of focusing operators like only with the set of focal alternatives (e.g. Rooth 1985). In our example, the two distinct questions (1a) and (1b) pose the same conditions on the focal structures of their answers and can have different effects on the quantificational domain of subsequent only. These two facts constitute a problem for these standard theories unless they come equipped with a smart analysis of the dynamics of domain restriction which plays a role in these cases.

Elegant dynamic analyses of questions have been developed in the tradition of the partition theory of Groenendijk and Stokhof 1984. In the partition theory, the meaning of a question is identified with the set of meanings of all its complete answers. In a dynamic setting, questions partition information states, and answers eliminate blocks of these partitioned contexts (see Groenendijk 1998-99, but also Jäger 1996 and Hulstijn 1997). These theories in which interrogatives and indicatives update a context, constitute a simple model of how information in discourse 
is organized by the question-answer relation (Carlson 1983, Roberts 1996). The notion of a partial or complete answer is specified in terms of entailment which is uniformly defined for indicative and interrogative sentences. Although logically very appealing, these theories are, empirically, not completely satisfactory. Partitions seem to be too coarse-grained for a proper treatment of focus and constituent answers. For example, Groenendijk 1999 cannot account for the different content expressed by a constituent answer like (2c) after (2a) and after (2b), for the two questions, having the same set of complete answers, induce exactly the same partition.

(2) a. Who smokes?

b. Who doesn't smoke?

c. $[\text { John }]_{F}$.

Related difficulties also arise for theories in the Hamblin/Karttunen/Rooth tradition, e.g. problems with multiple foci (Krifka 1992) and alternative questions (von Stechow 1991, Krifka 2001). The standard treatment of alternatives as sets of (propositional) answers is not fined-grained enough and for a proper account we need the abstracts underlying the questions (see also Ginzburg 1995 and van Rooy 1997) and direct access to focus, i.e. structured meanings.

In a structured meaning account we have fitting analyses of questions and focus: questions denote abstracts, $\lambda \vec{x} \phi$, i.e. functions that when applied to the meaning of the possible constituent answers yield the meaning of the corresponding full sentential answers; and focus leads to a partition of the semantic value of an expression into a background part, also a function, and a focus part: $\langle\lambda \vec{x} \phi, \vec{a}\rangle$. Although structured meanings seem to supply us with the right level of fine-grainedness, this account does not assume interrogatives to belong to a uniform category or semantic type and, therefore, lacks an elegant analysis of examples like (3) which involves the embedding and coordination of questions:

(3) Adam knows whether it's Mary's party, who will go and who invited whom.

In what follows we will extend Gawron's (1996) dynamic model of domain restriction with an analysis of interrogative sentences. Utilizing the close correspondence between information states in dynamic semantics - sets of world-assignment pairs - and structured propositions, the obtained analysis of questions and focus will solve the discussed difficulties combining the positive sides of partitions and structured meanings, and will allow a number of further applications, such as a promising account of topic marking in English.

\section{An update semantics of questions and focus}

In Gawron 1996, the introduction of a quantificational operator is separated by the introduction of the quantificational domain. The latter is allowed to be fixed non- 
locally. The intuition is that domains of quantification are constructed by combining constraints that arise from different sources. These constraints are encoded in so called environments which map variables to sets of possible assignments encoding information about which values are possible for them. We propose to interpret the semantic contribution of interrogative sentences in term of extensions of these Gawronian environments. In our formalism, an environment is a sequence of sets of world-assignment pairs. We will take these sets to represent the topics under discussion in the current context. Interrogative sentences will be analyzed as setting up new topics, or expanding on previously introduced ones.

From a topic in an environment we can easily recover the partition it would induce on the current information state. Therefore, we will be able to define all of the logical notions which are relevant for a theory of questions and answers. Since our topics are as fine-grained as abstracts, we will improve, though, on the partition theory with respect to phenomena like constituent answers or alternative questions. On the other hand, since interrogatives are associated with a uniform semantic type, we will also improve on the structured meaning account with respect to the embedding and coordination of questions. Finally, since, as in Gawron, topics encode domain restrictions, we will be able to account for the impact of questions on subsequent domains of alternatives and account for the 'gentlemen' example discussed in the introductory section of this article.

\subsection{A closer look}

The proposed semantics is an extension of Aloni, Beaver, Clark 1999 (built on Gawron 1996) with an explicit analysis of interrogative sentences. Zeevat 1994 and van Rooy 1997 defend similar ideas in somewhat different formalisms.

Formulae are associated with context change potentials. A context $s_{e}$ is a pair consisting of an information state $s$ (a set of world-assignment pairs) and an environment $e$ (a sequence of states). States encode what is known and what antecedents are available for future anaphora; environments encode information about what is merely under discussion. Contexts $s_{e}$ can be depicted as in (4) where each box stands for an information state.

(4) $\left\langle s: \square, e: \square_{1}, \ldots, \square_{n}\right\rangle$

For example, the empty box in (5a) stands for the state of minimal information, whereas the box in (5b) encodes the information that $x$ is $P$.

(5) a. $\square \mapsto\{(\emptyset, w) \mid w \in W\}$

(minimal information)

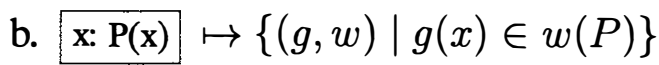

Questions set up (or expand on previously introduced) topics. Interrogative sentences are formed by prefixing a question mark and a sequence of variables $x_{1}, \ldots, x_{n}=\vec{x}$ to a formula. The effect of updating with sentence $? \vec{x} \phi$ is that the last element in the output environment is a state that verifies $\phi$.

A polar question like (6a), represented as in (6b), extends the environment with a state that entails that Mary smokes (e.g. (6c)). 
(6) a. Does Mary smoke?

b. ?S(m)

c. $\langle\square\rangle \llbracket ? S(m) \rrbracket\langle\square, \mathrm{S}(\mathrm{m})\rangle$

A constituent question like (7a) represented as (7b) extends the environment with a state which encodes the information that $x$ is a smoker (e.g. (7c)). Intuitively we can think of (7b) as introducing the set of smokers as topic under label $x$.

(7) a. Who smokes?

b. $? x S(x)$

c. $\langle\square\rangle \llbracket ? x S(x) \rrbracket\langle\square, \mathbf{x : \mathbf { S } ( \mathbf { x } ) ] \rangle}$

\section{Topics and sets of propositions}

From a topic in $s_{e}$ we can uniquely derive the corresponding Hamblin denotation ${ }^{1}$ or G\&S partition both expressed as a(n equivalence) relation over the current state $s$. As an illustration, consider the topics represented in (8b) and (9b) introduced by the questions (8a) and (9a). The partitions and Hamblin denotations induced by these topics can be depicted as in (8c) and (9c). Assume that $j$ and $m$ are the only individuals in the domain.

(8) a. $? x S(x)$

b. $\langle\square, \mathbf{x :} \mathbf{S}(\mathbf{x})\rangle$

c. Hamblin: $\frac{S(m)}{S(j)}$

G\&S:

\begin{tabular}{|c|}
\hline$\forall x \neg S(x)$ \\
\hline$\forall x(S(x) \leftrightarrow x=m)$ \\
\hline$\forall x(S(x) \leftrightarrow x=j)$ \\
\hline$\forall x(S(x) \leftrightarrow(x=j \vee x=m))$ \\
\hline
\end{tabular}

(9) a. ?S(m)

b. $\langle\square, \mathrm{S}(\mathrm{m})\rangle$

c. Hamblin: $S(m) \quad$ G\&S: $\frac{S(m)}{\frac{\neg S(m)}{i}}$

The state-environment pairs in (b) are more fine-grained than the G\&S partitioned states in (c). E.g. (9) after (8) does not add anything to the partition, but it extends the environment in a non-trivial way.

(10) a. $? x S(x) \wedge ? S(m)$

b. $\langle\square, \mathbf{x : S ( x ) , S ( m ) ~}\rangle \mapsto$ 
c.

\begin{tabular}{|c|}
\hline$\forall x \neg S(x)$ \\
\hline$\forall x(S(x) \leftrightarrow x=m)$ \\
\hline$\forall x(S(x) \leftrightarrow x=j)$ \\
\hline$\forall x(S(x) \leftrightarrow(x=j \vee x=m))$ \\
\hline
\end{tabular}

$\sqcap \frac{S(m)}{\square S(m)}=$\begin{tabular}{c|}
$\forall x \neg S(x)$ \\
\hline$\neg x(S(x) \leftrightarrow x=m)$ \\
\hline$\forall x(S(x) \leftrightarrow x=j)$ \\
\hline$\forall x(S(x) \leftrightarrow(x=j \vee x=m))$ \\
\hline
\end{tabular}

We will exploit these two levels of fine-grainedness in a crucial way. We will define the logical notion of entailment, $\models$, in terms of the partitioned states (exactly as in Groenendijk 1998-99), and the more discourse oriented notion of support, $\approx$, in terms of the more fine-grained state-environment pairs. As for indicative sentences, support and entailment are the same notion. But, they crucially differ with respect to questions.

A question is entailed iff its update does not further partition the input state. An interrogative ? $\vec{x} \psi$ is entailed after an indicative $\phi$ iff the indicative is a complete answer to $? \vec{x} \psi$. An interrogative ? $\vec{x} \psi$ is entailed after another interrogative ? $\vec{y} \phi$ iff any complete answer to ? $\vec{y} \phi$ is a complete answer to ? $\vec{x} \psi$. E.g.
a. $\forall x(S(x) \leftrightarrow x=m) \models ? x S(x)$,
but $S(m) \not \models ? x S(x)$
b. $? x S(x) \models ? S(m)$

On the other hand, a question is supported iff the topic it introduces is already entailed in the input context, either by the input state or by an old topic in the input environment. After an indicative $\phi$, interrogative ? $\vec{x} \psi$ is supported iff $\phi$ entails $\exists \vec{x} \psi$, that is, if $\phi$ entails a positive congruent answer to the question. After another interrogative ? $\vec{y} \phi$, sentence ? $\vec{x} \psi$ is supported iff $\exists \vec{y} \phi$ entails $\exists \vec{x} \psi$.

(12) a. $S(m) \approx ? x S(x)$

b. ?S(m) $\approx ? x S(x), \quad$ but $? x S(x) \not 2 ? S(m)$

Entailment seems to be relevant for indirect uses of interrogatives. The sentences (13a-b) are valid implications, but (13c) is not.

(13) a. If John knows that only Mary smokes, then John knows who smokes.

b. If John knows who smokes, then John knows whether Mary smokes.

c. If John knows that Mary smokes, then John knows who smokes.

As we will see, support is relevant for direct uses of questions in discourse and will play a crucial role for our characterization of focus and its pragmatic role.

\section{Topics and quantification}

Topics encode domain restrictions. An update with a quantified sentence $\exists x \phi$ only modifies the state parameter but crucially depends on the environment parameter, in particular on the last topic in which the quantified variable is defined, $e(x)$, which encodes all restrictions previously placed on $x$. 
(14) a. ? $x S(x) \wedge \exists x P(x)$

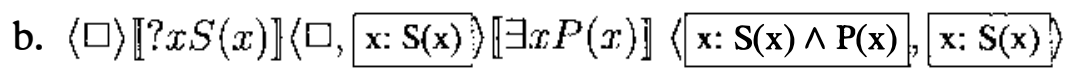

The valid entailment in (15) illustrate a crucial feature of our formalism. Questions can restrict subsequent quantification if coindexed.

(15) $? \vec{x} \phi_{1} \wedge \ldots \wedge ? \vec{x} \phi_{n} \wedge \exists \vec{x} \psi \models \exists \vec{x}\left(\left(\phi_{1} \wedge \ldots \wedge \phi_{n}\right) \wedge \psi\right)$

\section{Presupposition}

Topics can be crucially presupposed. Presupposition (denoted by Beaver's $\partial$ ) expresses conditions on the input context which must be satisfied for the sentence to be defined (Stalnaker, Heim, Beaver). An update with a presupposition $\partial \phi$ is defined in $s_{e}$ iff $s_{e}$ supports $\phi$.

Note that presupposition is defined in terms of support rather than entailment. This means that a presupposed topic like $\partial[? x I(a, x)]$ is defined after $I(a, b)$, but not after ?xyI $(y, x)$. This notion of presupposition will play a crucial role for our treatment of focus.

\section{Focus}

Focus indicates the presence of a topic in the context. More specifically, as in the structured meaning approach, focus leads to a 'partition' of the sentence into: (1) a presupposed topic (background); and (ii) an existential sentence (focus).

A sentence like (16a) represented as (16b) presupposes that the set $S$ of smokers is under discussion and asserts that Mary is part of it.

(16) a. $[\text { Mary }]_{F}$ smokes.

b. $\partial[? x S(x)] \wedge \exists x(x=m)$

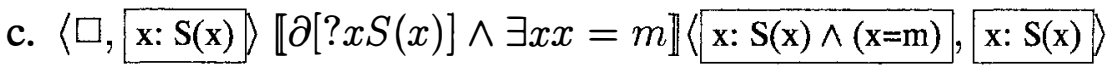

This analysis covers focus in questions as illustrated in (17). Question (17a) represented in (17b) again presupposes that the set of smokers is under discussion, and asks whether Mary is among them.
a. Does $[\text { Mary }]_{F}$ smoke?
b. $?(\partial[? x S(x)] \wedge \exists x(x=m))$

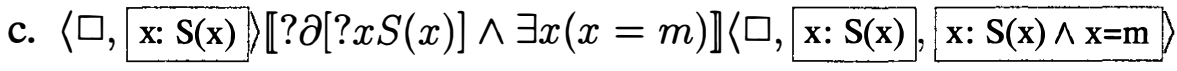

Note that from the representations in (16b) and (17b) we can recover the ordinary meanings of the sentences, $(16 \mathrm{~b}) \models S(m)$, and $(17 \mathrm{~b}) \models ? S(m)$. 


\section{Applications}

In this section, we will show how the formalism presented in the previous section allows us to solve the problems discussed in the introductory part of the present article. Let us first briefly present our analysis of which-interrogatives, which will play a role in some of the following applications.

\subsection{Which-questions}

We assume that a which-phrase gives rise to the presupposition that the set over which it ranges is already given as a topic. Questions (18a) and (19a) are represented as in (18b) and (19b).

(18) a. Which men are bachelors?

b. $\partial[? x M(x)] \wedge ? x B(x)$

(19) a. Which bachelors are men?

b. $\partial[? x B(x)] \wedge ? x M(x))$

Question (18) presupposes that the set of men is under discussion and it asks which of them are bachelors. Question (19) presupposes that the set of bachelors is under discussion and it asks which of them are men. In distinction with Groenendijk \& Stokhof's (1984) treatment, according to which (18) and (19) are equivalent, this analysis allows us to capture the contrast between these two questions. Although (18b) and (19b) determine the same partition, under the assumption that in all worlds all bachelors are men, $(19 \mathrm{~b})$ is vacuous whenever defined. In distinction with (18b), which is not a trivial question.

\subsection{Association with focus: only}

We treat the focusing operator only as an indexed sentential operator only ${ }_{\vec{x}}$, where $\vec{x}=x_{1}, . ., x_{n}$ are focused variables (see Aloni et al 1999). The interpretation of only $_{\vec{x}}$ involves a universal quantification over the focused variable $\vec{x}$ which is automatically restricted by the presupposition expressed by focus. As an illustration consider sentence $(20 \mathrm{a})$ represented as in (20b):

(20) a. John only introduced $[\text { Bill }]_{\mathrm{F}}$ to Sue.

b. $\operatorname{only}_{x}(\partial[? x I(j, x, s)] \wedge \exists x(x=b))$

As in standard analyses of (un)selective binding, only ${ }_{x}$ changes the quantificational force of the quantifier binding $x$ from existential to universal. Given the presupposition expressed by focus, this universal quantification is automatically restricted to individuals John introduced to Sue. In a minimal context satisfying the presupposition of the sentence, we predict for (20a) the expected meaning 'The only individual John introduced to Sue is Bill', as in the standard analyses of this phenomenon, $(20 \mathrm{~b}) \models \forall y(I(j, y, s) \leftrightarrow y=b) .^{2}$

In distinction with the standard analyses, however, we have a systematic account of other restrictions on the quantificational domain of only which can arise from sources other then focus, for example a preceding question like (21a). 
(21) a. Which gentleman did John introduce to Sue?

b. $\partial[? x G(x)] \wedge ? x I(j, x, s)$

c. John only introduced $[\text { Bill }]_{F}$ to Sue.

d. only $_{x}(\partial[? x I(j, x, s)] \wedge \exists x(x=b))$

After (21a), (21c) can obtain the weaker interpretation 'The only gentleman John introduced to Sue is Bill', (21b), (21d) $\models \forall y((G(y) \wedge I(j, y, s)) \leftrightarrow y=b)$.

\subsection{Congruence}

In this section we will show how our dynamic analysis enables us to give an interesting characterization of the notion of discourse congruence which covers contextual restrictions while avoiding problems of over- and under-focus, and uniformly applies to answers, questions, strategies and denials.

In our proposal a sentence $\phi$ is congruent after $\psi$ iff(i) the presupposition of $\phi$ is defined after $\psi$, and (ii) no more material is in focus than needed to satisfy (i).

Our conditions (i) and (ii) are closely related to Schwarzschild's (1999) givenness and avoid focus constraints. As Schwarzschild's givenness, condition (i) is a formalization of the traditional idea that non-focused material must be old. In distinction with Schwarzschild, however, our analysis of givenness is of a rather global nature: the existential closure of the non-focused parts of a whole clause has to 'given' in the context, not the individual words themselves. ${ }^{3}$ Condition (ii) corresponds to Schwarzschild's optimality theoretic constraint to avoid unnecessary focus: in our framework it will prevent us from placing more material in focus than is strictly necessary to allow the context to support the focal presupposition of the sentence.

Focus presupposes a question and presupposition is defined in term of support. Therefore in order to understand condition (i) it is important to recall after which sentences a question is supported. As noted above, and explained more formally in the appendix, a question ? $\vec{x} \psi$ is supported after an indicative $\phi$ or interrogative $? \vec{x} \phi$ iff $\phi$ or $\exists \vec{x} \phi$ entails $\exists \vec{x} \psi$. By this notion of support, we can account for the intuition that a sentence is congruent because it either 'matches' the question the sentence addresses example (22), or it stands in contrast with an earlier made assertion (example (23)).
a. Who voted for Mary?
$? x V^{*}(x, m)$
b. $[\mathrm{John}]_{\mathrm{F}}$ voted for Mary.
$\partial[? x V(x, m)] \wedge \exists x(x=j)$

(23)
a. Bill voted for Mary.
$V(b, m)$
b. No, [John $]_{F}$ voted for Mary.
$\partial[? x V(x, m)] \wedge \exists x(x=j)$ 
Sentence (22b) and (23b) are congruent after (22a) or (23a), because both (b) sentences are minimally focused to be defined after the respective antecedents. In the same context, the alternative focus structures in (24) are predicted to be infelicitous. Sentence (24a) is undefined after (22a) or (23a). Had John not been in focus in (24b), then the presupposition of the sentence would already be supported after (22a) or (23a).

(24) a. ${ }^{*}$ John voted for [Mary $]_{\mathrm{F}} . \quad \partial[? x V(j, x)] \wedge \exists x(x=m)$

b. $*[\mathrm{John}]_{\mathrm{F}}$ voted for [Mary $]_{\mathrm{F}} . \quad \partial[? x y V(x, y)] \wedge \exists x(x=j) \wedge \exists y(y=m)$

Just like Roberts 1996, our notion of congruence applies to questions and question strategies as well. Also in this case, underfocused questions will be undefined and overfocused ones will violate our minimality constraint.

Finally, our dynamic analysis also immediately predicts correctly for sequences in which contextual restrictions play a crucial role. Since question (25) supports question (22a), in our analysis the two questions pose the same condition on the focal structure of their answers.

(25) Which Democrats voted for Mary? $\quad \partial[? x D(x)] \wedge ? x V(x, m)$

\subsection{Constituent Answers}

A constituent answer is expressed as an existential sentence, the domain of which is crucially restricted by the preceding question.

The following analysis of yes-no answers is based on Dekker 2002 which also has a treatment of plural and quantified answers. Yes and no are represented as in (26d):
a. Does Mary smoke?
b. ?S(m)
c. $[\mathrm{Yes}]_{\mathrm{F}} /[\mathrm{No}]_{\mathrm{F}}$.
d. $\exists \top / \neg \exists \top$

Given these representations we correctly predict that after (26a), yes means 'Mary smokes': (26b), $\exists \top \models S(m)$; and no means 'Mary does not smoke': (26b), $\neg \exists \top \models$ $\neg S(m)$.

Term answers like John are represented as in (27f).
a. Who smokes?
b. $? x S(x)$
c. Who doesn't smoke?
d. $? x \neg S(x)$
e. $[\mathrm{John}]_{\mathrm{F}}$.
f. $\exists x(x=j)$

After (27a), John means 'John smokes': (27b), (27f) $\models S(j)$; after (27c), it means 'John does not smoke': (27d), (27f) $\models \neg S(j)$. 


\subsection{Alternative questions}

In this section we discuss the contrast between polar and alternative questions. Proposition set theories of questions in both the G\&S and Hamblin/Karttunen traditions have problems in accounting for alternative readings of questions as argued in von Stechow 1991 and Krifka 2001. We would like to show that our analysis is fine-grained enough to express both question readings.

Question (28) is ambiguous between a polar question reading (expected answers: yes/no) and an alternative question reading (expected answers: tea/coffe).

(28) Do you want coffee or tea?

Intonation seems to play a disambiguating role. In alternative questions, the alternatives are stressed.

(29) Do you want COFFEE or TEA?

a. *Yes / *No. b. Coffee / Tea.

If we assume for (29) the focal structure in (31a), the contrast between polar and alternative readings follows directly from our analysis of focus.

(30) a. [Do you want coffee or tea $]_{F}$ ?

(polar)

b. ?(W(c) $\vee W(t))$

c. Yes / No.

d. $\exists \top / \neg \exists \top$

e. topic: $\mathbf{W}(\mathrm{c}) \mathrm{VW}(\mathrm{t}) \quad \mathrm{f}$. Hamblin: You want coffee or tea

(31) a. Do you want $[\text { coffee }]_{F}$ or $[\text { tea }]_{F}$ ?

(alternative)

b. $?(\partial[? x W(x)] \wedge \exists x x=c \vee x=t)$

c. Coffee / Tea.

d. $\exists x(x=c) / \exists x(x=t)$

e. topic: $\mathrm{x: \textrm {W } ( \mathrm { x } ) \wedge ( \mathrm { x } = \mathrm { c } \vee \mathrm { x } = \mathrm { t } )} \mapsto \mathrm{f}$. Hamblin: $\begin{gathered}\text { You want coffee } \\ \text { You want tea }\end{gathered}$

The formulae (30b) and (31b) set up different topics, therefore (i) they express different questions (compare the Hamblin denotation in (f) induced by the introduced topic in (e)); and (ii) they allow different constituent answers. 


\subsection{Embedded questions}

In the introduction we pointed out that although the fine-grainedness of the structured meaning analysis of questions is needed to account for constituent answers and alternative questions, it is problematic too. By assuming that different types of interrogatives have denotations of different categories, the structured meaning account has problems with the coordination and embedding of questions. This problem disappears once one assumes a propositional set theory as those proposed by Hamblin, Karttunen or Groenendijk \& Stokhof. According to these latter theories, polar and (multiple) $w h$-questions all have denotations of the same category, and all these questions can thus be coordinated under know and wonder as in (32):

(32) Adam knows/wonders whether it's Mary's party, who is invited, and who will kiss whom.

Only Groenendijk \& Stokhof's analysis, however, correctly predicts that indicatives can also be freely coordinated under know with interrogatives:

(33) Adam knows that it's Mary birthday and who is invited to come.

Moreover, by thinking of the denotation of a question as an equivalence relation, the inclusion relation accounts for entailment not only in case of declaratives, but also for interrogatives. Our approach shares with Groenendijk \& Stokhof these desirable consequences. First, coordination between indicatives and interrogatives of any 'type' is unproblematic: a context $s_{e}$ can also be updated with $\phi$ if $\phi$ contains both an indicative and an interrogative. This updated context gives rise to a structured state: the partition $P\left(s_{e}[\phi]\right)$. As shown in the appendix, entailment can be defined in terms of subsistence between such structured states. Taking $K_{a}(i)$ to denote the epistemically accessible worlds to Adam in possibility $i$, and ignoring anaphoric dependencies and presuppositions, we can simply assume that the stateenvironment pair with respect to which the embedded clause should be interpreted in possibility $i=\langle g, w\rangle$ is $K_{a}^{*}(i)=\left\{\langle h, w\rangle: h=g \wedge v \in K_{a}(w)\right\}_{e_{0}}$, where $e_{0}$ is the 'empty' environment which makes $P\left(K_{a}^{*}(i)\right)=\left\{\left\langle j, j^{\prime}\right\rangle: j, j^{\prime} \in K_{a}^{*}(i)\right\}$. Now we can define the update of context $s_{e}$ with sentence 'know $(a, \phi)$ ' as follows:

$$
\text { (34) } s_{e}[k n o w(a, \phi)]=\left\{i \in s: K_{a}^{*}(i) \text { entails } \phi\right\}_{e}
$$

This has the result that sentence (33), for instance, is predicted to be true in possibility $i=\langle g, w\rangle$ iff (i) Adam knows thąt it's Mary's birthday, and (ii) Adam knows that $d$ is invited to come if and only if $d$ is actually invited in $w$, for every $d .{ }^{4}$

Groenendijk \& Stokhof (1982) account for the fact that to wonder, in distinction with to know, cannot embed indicatives by assuming that the former verb is intensional and not extensional. We won't make use of this assumption, however. Instead, we will assume that a sentence of the form ' $w o n d e r(a, \phi)$ ' can only be true in $i$ if (i) $K_{a}^{*}(i)$ does not entail $\phi$, but (ii) $\phi$ does not eliminate any possibilities of $K_{a}^{*}(i)$. This has the result that $\phi$ cannot be an indicative, because that would either eliminate possibilities, or else be entailed by $K_{a}^{*}(i)$. 


\subsection{Relevance}

In this section, we extend Groenendijk's (1999) notion of relevance to questions. In doing so, we propose a formalization of Roberts's (1996) insight that a question is relevant iff it is part of a strategy to answer the immediate question under discussion. ${ }^{5}$

Groenendijk 1999 proposes the following characterization of the notion of a relevant (or pertinent, coherent) move in a discourse:

(35) A move is relevant iff it is (i) about the issue under discussion; (ii) non vacuous; and (iii) consistent.

Groenendijk's characterization of (i) in terms of licensing ${ }^{6}$ and (ii) in terms of entailment prevents a correct application of this notion to questions. According to Groenendijk, questions are always licensed, and are informative iff they are not entailed. Therefore, we obtain the predictions in (36), which are highly counterintuitive.

(36) a. Who smokes?

b. Well, does Mary smoke?

(not relevant)

c. Well, does Mary work?

(relevant)

Question (b) is not relevant after (a) because, since it is entailed, it is not informative. Question (c) is relevant because licensed, not entailed and consistent. Intuitively though, both questions are non-vacuous moves after (a), but only (b) is about (a), since, intuitively, it suggests a strategy to answer (a). Entailment does not seem to be the right notion to characterize non-vacuous questions, and Groenendijk's licensing should be modified to capture aboutness of questions, and not only of assertions.

We propose to define informativity in terms of support rather than entailment and to generalize Groenendijk's notion of licensing as follows. Let $\phi$ ? be $\phi$, if $\phi$ is an interrogative, ? $\phi$ otherwise. A sentence $\phi$ is licensed iff $\phi$ ? is entailed. Intuitively, a sentence is licensed iff it exclusively addresses the question under discussion $Q$ either by giving a partial answer to $Q$ (as in Groenendijk 1999) or by introducing a question the answers of which are partial answers to $Q$, i.e. an entailed question.

The obtained notion of relevance gives us the correct predictions in (37).

(37) a. Who smokes?

b. Well, does Mary smoke?

(relevant)

c. Mary smokes. (relevant)

d. Well, does Mary work?

(not relevant)

Sub-question (37b) is licensed, but not supported (although entailed) after (37a), therefore it is relevant, as well as sentence (37c). Question (37d) is not relevant because it is not licensed. 


\subsection{Topic}

In the literature, there exist two popular views on what a sentence is about. According to a tradition starting with Paul 1880, the topic of a sentence is the question the sentence is addressing. According to another tradition going back at least to Goodman 1961, the topic of a sentence is the referent the sentence is about. In more recent analyses along the second tradition, e.g. Reinhart 1981 and Vallduví 1990 , this referent need not be a particular real entity, but is thought of rather as a discourse referent. By representing questions as discourse referents in an environment, we suggest that these two views are two sides of the same coin.

What a sentence is about is also linguistically marked, in English, by the use of accent.

(38) a. Who ate what? What about Fred? What did he eat?

b. Fred ${ }_{B}$ ate the beans ${ }_{A}$.

According to Jackendoff 1972, the rising $A$ accent marks dependent focus, while the falling $B$ accent marks independent focus. According to our analysis, focus presupposes a question: it indicates that it addresses a certain question. Because two foci are used, it presupposes at least the multiple wh-question (39a) as in Roberts 1996. However, as in Büring 1999, we will also assume that (38b) presupposes (39b).
a. Who ate what?
?xyAte $(x, y)$
b. What did Fred eat?
?yAte $(f, y)$

According to Roberts 1996, the two questions form part of a questioning strategy. Our notion of relevance between questions shows that the question (39b) can be part of a strategy to answer (39a), but not the other way around. Thus, we can determine that the presuppositions and assertion of $(38 \mathrm{~b})$ should be represented as follows:

(40) a. Fred $_{B}$ ate the beans .
b. $\partial[? x y \operatorname{Ate}(x, y)] \wedge \partial[? y \operatorname{Ate}(f, y)] \wedge \exists y[y=b] \quad$ or equivalently
c. $\partial[? x y \operatorname{Ate}(x, y)] \wedge \partial[? x(x=f)] \wedge \exists y[y=b]$

The ordinary meaning of the sentence is entailed: $(40 \mathrm{c}) \models \operatorname{Ate}(f, b)$.

What Jackendoff called $A$ and $B$ accęnt is called focal and topical accent respectively by Büring 1999 . Büring proposes that a sentence like (38b) not only has a focal-value, but also a topic-value. The former corresponds with our question (39b), but the latter is not a question, but rather a set of questions: for each relevant individual $d$ the question what $d$ ate. To account for the intuition that (38b) is only a partial answer to question (39a), he states an extra disputability condition. If we denote the topic-value of $A$ by $[[A]]^{t}$, the condition says that if in $A$ a topical accent is used, at least one question in $[[A]]^{t}$ must still be open. This disputability condition, however, gives rise to the so-called last answer problem. 
(41) a. Who ate what?

b. Mary $_{B}$ ate sprouts $A$, and

c. Fred $_{B}$ ate the beans ${ }_{A}$.

After (41b) is given, answer (41c) might resolve the whole question (41a), which is in conflict with Büring's disputability condition. We have taken over Roberts' (1996) suggestion that 'topic'-accent indicates, or presupposes, the use of a certain questioning strategy: (38b) presupposes both (39a) and (39b), and congruence demands that the former must have been asked before the latter. But note that from our relevance condition we can still derive Büring's disputability in case (38b) is used out of context without making use of non-ordinary semantic values. The reason is that the assertion presupposes questions (39a) and (39b), and that our relevance condition on questions demands that (39b) can only be part of a strategy to answer (39a) in case there is at least one individual different from Fred whose eating behavior is still in question.

Büring 1999 makes crucial use of his disputability condition to explain why sentence (42a) only has a $\neg \forall$ reading, i.e., that (42a) cannot mean (42b):

(42) a. $\mathrm{All}_{B}$ politicians are not n $_{\text {corrupt. }}$

b. $\forall x[\operatorname{Pol}(x) \rightarrow \neg \operatorname{Crpt}(x)]$

However, this much follows already from our assumption that sentences with independent and dependent focus presuppose two questions, and the general condition that question $Q^{\prime}$ cannot be part of a strategy to answer $Q$ if they denote the same partition. Notice that it follows from our reasoning above that (42a) presupposes either $\partial[? x \operatorname{Pol}(x)] \wedge ? x \operatorname{Crpt}(x)$ and (43a), or $\partial[? x \operatorname{Pol}(x)] \wedge ? x \operatorname{Crpt}(x)$ and (43b):
a. $\frac{\forall x[\operatorname{Pol}(x) \rightarrow \operatorname{Crpt}(x)]}{\neg \forall x[\operatorname{Pol}(x) \rightarrow \operatorname{Crpt}(x)]}$
b. \begin{tabular}{c}
$\forall x[\operatorname{Pol}(x) \rightarrow \operatorname{Crpt}(x)]$ \\
\hline$\forall x[\operatorname{Pol}(x) \rightarrow \neg \operatorname{Crpt}(x)]$ \\
\hline
\end{tabular}

Now suppose that (42a) actually presupposed (43b). Assuming that the presupposition of a question is the union of its possible answers, it follows that (42a) must presuppose that either all politicians are corrupt, or no politician is corrupt. Assuming that question $Q^{\prime}$ can only be part of a strategy to resolve 'goal'-question $Q$ if $Q^{\prime}$ and $Q$ do not denote the same partition, we demand that partition (43b) is not the same partition as the one denoted by $\partial[? x \operatorname{Pol}(x)] \wedge ? x \operatorname{Crpt}(x)$. This means that there must be at least more than one politician, and that it is not presupposed that either all politicians are corrupt, or that none of them is corrupt. So, our conditions demand that the partition due to $\partial[? x \operatorname{Pol}(x)] \wedge ? x \operatorname{Crpt}(x)$ denotes a cell where some but not all politicians are corrupt. But this is inconsistent with the presupposition of (43b), which rules out the possibility that (42a) presupposes (43b). The 
sequence consisting of $\partial[? x \operatorname{Pol}(x)] \wedge ? x \operatorname{Crpt}(x)$ and (43a), on the other hand, is predicted to be appropriate, and will thus be chosen. But this means that (42a) can be given only as answer to (43a), and thus can receive the $\neg \forall$ reading only.

\section{Conclusion}

We have analyzed within dynamic semantics how questions can restrict the domain of quantificational sentences used later in a discourse. We have done this by extending Gawron's (1996) dynamic analysis of domain restriction with questions. Our analysis of questions incorporates Groenendijk's (1999) logic of interrogation, but improves on it by introducing (basically) the abstracts underlying the questions to the discourse. In this way we are able to account for the context-dependent meaning of constituent-answers, free focus, association with focus and how the domain of focus sensitive operators like only can be determined by a previous question.

\section{Appendix}

\section{Formal Definitions}

The vocabulary of our language is like that of standard first-order predicate logic with identity, but with a polyadic existential quantifier $\exists x_{1}, \ldots, x_{n}$, and with the addition of with a sentential operator only $x_{x_{1}, . ., x_{n}}$, a presupposition operator $\partial$ and a question operator $? x_{1}, \ldots, x_{n}$. We do not have compound interrogatives or quantification into questions, but we have presupposed questions and can form sequences of questions (and assertions). As for the semantics, formulae are associated with context change potentials. A context $s_{e}$ is a pair consisting of an environment $e$ and an information state $s$. An information state consists of a set of world-assignment pairs. An environments is a sequence of information states. If $c=s_{e}$ is a context, then $S(c)=s$ and $E(c)=e$.

Elements of a state are called possibilities, given a possibility $i=\langle w, g\rangle$, we will write $i(\alpha)$ to refer to the denotation of $\alpha$ with respect to $g_{i}$ and $w_{i}$. As in Dekker (1993), possibilities are ordered by an extension relation $\prec: j$ extends $i$,

$i \prec j$ iff $w_{i}=w_{j} \& g_{i} \subseteq g_{j}$. This extension relation carries over to an ordering relation between information states: $s$ is a substate of $t, s \prec t$ iff $\forall i \in s: i \prec t$, where $i \prec t$ iff $\exists j \in t: i \prec j$.

Now we can give a recursive definition of the context-change potential of the formulae of the language. The basic formulae are defined as expected: they can only influence the state parameter $s$ and eliminate possibilities in $s$ in which the formulae are false:

1. $s_{e}\left[P t_{1}, \ldots, t_{n}\right]=\left\{i \in s \mid<i\left(t_{1}\right), \ldots, i\left(t_{n}\right)>\in i(P)\right\}_{e}$

In the interpretation rule of negation, we make crucial use of the ordering relation $\prec$. Just like atomic formulae, negation influences only the state parameter: 
2. $s_{e}[\neg \phi]=\left\{i \in s \mid i \nprec S\left(s_{e}[\phi]\right)\right\}_{e}$

Conjunction is defined as standard in dynamic semantics as sequential update:

3. $s_{e}[\phi \wedge \psi]=s_{e}[\phi][\psi]$

Until now the environments played virtually no role. They are crucial, however, for the semantic analysis of quantified sentences. The update of context $s_{e}$ with an existential sentence $\exists x_{1}, \ldots, x_{n} \phi$ is defined in terms the merge of two information states. The merging of information state $s$ with information state $s^{\prime}, s \wedge s^{\prime}$, is defined as the 'least upper bound' of $s$ and $s$ ' (see Dekker 1993):

$$
s \wedge s^{\prime}=\left\{i \mid \exists j \in s: \exists j^{\prime} \in s^{\prime}: \operatorname{dom}(i)=\operatorname{dom}(j) \cup \operatorname{dom}\left(j^{\prime}\right) \& j \prec i \& j^{\prime} \prec i\right\}
$$

If we define random assignment, $s[x]$, as $\{\langle w, g[x / d]\rangle:\langle w, g\rangle \in s \& d \in D\}$, we can define the update of $s_{e}$ with an existential sentence in terms of this mergeoperator as follows. Assume $x_{1}, \ldots, x_{n}=\vec{x}$ are not defined in $s .{ }^{7}$

$$
\text { 4. } s_{e}[\exists \vec{x} \phi]=\left(S\left(\left(s\left[x_{1}\right], \ldots,\left[x_{n}\right]\right)_{e}[\phi]\right) \wedge e(\vec{x})\right)_{e}
$$

where $e\left(x_{1}, \ldots, x_{n}\right)$ is the last state in $e$ in which the variables $x_{1}, \ldots, x_{n}$ are defined. More formally, if $e=\left\langle e_{1}, \ldots, e_{m}\right\rangle$, then (i) $e\left(x_{1}, \ldots, x_{m}\right)=e_{m}$, if $n=0$; (ii) $e\left(x_{1}, \ldots, x_{n}\right)=e_{i}$ in $e$, such that $x_{1}, \ldots, x_{n} \in \operatorname{dom}\left(e_{i}\right)$ and $\forall e_{j}\left[x_{1}, \ldots, x_{n} \in\right.$ $\left.\operatorname{dom}\left(e_{j}\right) \rightarrow j \leq i\right]$, otherwise.

Quantificational sentences make use of the environment, but have no influence on these environments themselves. Only questions have. The effect of updating context $s_{e}$ with question ? $\vec{x} \phi$ is that the last element in the new environment is a set of possibilities that verify $\phi$. If $e=\left\langle e_{1}, \ldots, e_{n}\right\rangle$ and $e^{\prime}=\left\langle e_{1}^{\prime}, \ldots, e_{m}^{\prime}\right\rangle$ are environments, then $e+e^{\prime}=\left\langle e_{1}, \ldots, e_{n}, e_{1}^{\prime}, \ldots, e_{m}^{\prime}\right\rangle$.

$$
\text { 5. } s_{e}[? \vec{x} \phi]=s_{e^{\prime}} \quad \text { where } e^{\prime}=e+S\left(s_{e}[\exists \vec{x} \phi]\right) \text {. }
$$

An update with a quantifier or a question will depend on the last introduced state in the current environment in which the quantified variables are defined. Yes-no questions and answers will depend on the last introduced state.

Finally, we define the operator only $\vec{x}$ which is analyzed as an asymmetric adverb of quantification (see Dekker 1993). Let $j \prec_{\vec{x}} i$ iff $j \prec i$ and $\operatorname{dom}\left(g_{i}\right)=$ $\operatorname{dom}\left(g_{j}\right) \cup\{\vec{x}\}$. Let $\phi$ be of the form $\partial\left[? \vec{x} \psi_{1}\right] \wedge \exists \vec{x} \psi_{2}$

6. $s_{e}\left[\operatorname{only}_{\vec{x}}(\phi)\right]=\left\{j \in s \mid\left\{i \mid j \prec_{\vec{x}} i \& i \prec S\left(s_{e}\left[\exists \vec{x} \psi_{1}\right]\right)\right\} \subseteq\{i \mid i \prec\right.$ $\left.\left.S\left(s_{e}[\phi]\right)\right\}\right\}_{e}$

Disjunction, implication and universal quantifier are defined as standard in terms of conjunction, negation existential quantifier. 
Topic and sets of propositions

From a topic $e_{k}$ of domain $x_{1}, \ldots, x_{n}=\vec{x}$ in a context $s_{e}$ we can derive the corresponding Hamblin denotation, $H_{k}^{s_{e}}$, or G\&S partition, $P_{k}^{s_{e}}$, both expressed as a(n equivalence) relation over $s$.

Definition 1 [Hamblin denotation]

$$
H_{k}^{s_{e}}=\left\{\langle i, j\rangle \mid i, j \in s \& \exists \vec{d} \in D^{n}: i[\vec{x} / \vec{d}] \prec\left(s \wedge e_{k}\right) \& j[\vec{x} / \vec{d}] \prec\left(s \wedge e_{k}\right)\right\}
$$

Definition 2 [G\&S partition]

$$
P_{k}^{s_{e}}=\left\{\langle i, j\rangle \mid i, j \in s \& \forall \vec{d} \in D^{n}: i[\vec{x} / \vec{d}] \prec\left(s \wedge e_{k}\right) \leftrightarrow j[\vec{x} / \vec{d}] \prec\left(s \wedge e_{k}\right)\right\}
$$

\section{Entailment and Support}

Building on Groenendijk (1998, 1999), we define entailment in term of subsistence between structured states. By $P\left(s_{e}\right)$ we will denote the partition induced on $s$ by all the topics in $e$. Let $L(e)$ be the length of $e$, i.e. if $e=\left\langle e_{1}, \ldots, e_{m}\right\rangle$, then $L(e)=m$.

Definition $3 P\left(s_{e}\right)=\bigcap_{k \in L(e)}\left(P_{k}^{s_{e}}\right)$

Partitions $P\left(s_{e}\right)$ assigned to contexts $s_{e}$ are equivalent to the structured states $\sigma$ defined in Groenendijk 1998. We denote by $\iota$ the pair $\langle i, j\rangle$ of world-assignment pairs elements of such a structured states. Groenendijk defines subsistence between structured states in terms of the notion of $\prec$ between world-assignment pairs defined above. A pair $\langle i, j\rangle$ subsists in $\left\langle i^{\prime}, j^{\prime}\right\rangle,\langle i, j\rangle \prec\left\langle i^{\prime}, j^{\prime}\right\rangle$ iff $i \prec i^{\prime} \& j \prec j^{\prime}$. This relation between pairs of possibilities carries over to a relation between structured states: $\sigma \prec \sigma^{\prime}$ iff $\forall \iota \in \sigma: \iota \prec \sigma^{\prime}$, where $\iota \prec \sigma^{\prime}$ iff $\exists \iota^{\prime} \in \sigma^{\prime}: \iota \prec \iota^{\prime}$.

We can now define entailment. We denote by $\min _{\phi}$ the context of minimal information in which an update with $\phi$ is defined.

Definition 4 [Entailment]

(i) $s_{e} \models \phi$ iff $P\left(s_{e}\right) \prec P\left(s_{e}[\phi]\right)$

(ii) $\phi_{1}, \ldots \phi_{n} \models \psi$ iff $\min _{\phi_{1}, \ldots, \phi_{n}, \psi}[\phi] \models \psi$

Support is defined in terms of subsistence between contexts, rather than partitioned states. A context $s_{e}$ subsists in context $t_{f}, s_{e} \prec t_{f}$ iff $s \prec t$ and $e \prec f$, where an environment $e$ subsists in $f, e \prec f$, iff $\forall f_{j} \in f: \exists e_{i} \in e: e_{i} \prec f_{j}$.

Definition 5 [Support]

(i) $s_{e} \approx \phi$ iff $s_{e+s} \prec s_{e+s}[\phi]$

(ii) $\phi_{1}, \ldots, \phi_{n} \approx \psi$ iff $\min _{\phi_{1}, \ldots, \phi_{n}, \psi} \approx \psi$

In terms of support, we define Beaver's (1995) presupposition operator.

Definition 6 [Presupposition]

$$
s_{e}[\partial \phi]=s_{e^{\prime}}^{\prime} \text { iff } s_{e}[\phi]=s_{e^{\prime}}^{\prime} \& s_{e} \approx \phi, \text { undefined otherwise. }
$$




\section{Endnotes}

* Part of this material is joint work with David Beaver, Brady Clark. Special thanks to Paul Dekker for insightful discussions and to Darrin Hindsill for enhancing our English.

${ }^{1}$ Just like Roberts 1996, we also call the Hamblin denotation of a question not the set of its congruent answers, but the set of its questioned propositions. For this notion, we do not have to assume that the denotation of polar questions is determined differently from the denotation of (multiple) wh-questions. Standard Hamblin denotations for polar questions can also be derived, but, in our view, are less interesting.

${ }^{2}$ As in the structured meaning theory, we have direct access to the focused value. Therefore a number of examples which constitute a problem for Rooth's alternative semantics, are unproblematic in the present framework. For example we can express association with multiple foci (Krifka 1992), and we avoid Rooth's problem of intensionality (Rooth 1985 ch. 2 footnote 13). Sentence Nine is only the square of [three $]_{F}$ expresses a falsehood in our analysis.

${ }^{3}$ Our analysis also has nothing to say about embedded $F$-marking and de-accenting.

${ }^{4}$ Of course, to account for focus in the embedded clause, we might assume a more interesting interaction between $K_{a}(i)$ and the environment of the 'main' context.

${ }^{5}$ Eventually our characterization of the notion of a strategy of inquiries should take into account the average informativity of the possible answers, or borrowing a term from information theory, the entropy of the related questions (see van Rooy 2000). This would allow us distinguish sub-question (b) from (c) in example (44). The former is intuitively part of a much more efficient strategy to answer (a).

(44) a. Who ate what?

b. What did Fred eat?

c. Did Fred and Mary eat the beans?

${ }^{6}$ Groenendijk's licensing turns out to be equivalent to Lewis's (1988) notion of aboutness.

${ }^{7}$ As in Heim 1983, variables cannot be reset. So, in addition to formulae containing free variables, quantified sentences are partial updates as well. Since this issue is not directly relevant to the issues discussed in this article, we will pass over it in what follows.

\section{Bibliography}

Aloni, M., Beaver, D., and Clark, B.: 1999, Topic and focus sensitive operators, in P. Dekker (ed.), Proceedings of the Twelfth Amsterdam Colloquium, ILLC, University of Amsterdam, Amsterdam

Beaver, D.: 1995, Presupposition and Assertion in Dynamic Semantics, Ph.D. thesis, CCS, Edinburgh

Büring, D.: 1999, Topic, in P. Bosch and R. van der Sandt (eds.), Focus - Linguistic, Cognitive, and Computational Perspectives, pp 142-65, Cambridge University Press 
Carlson, L.: 1983, Dialogue Games: An Approach to Discourse Analysis, Synthese Library, Reidel

Dekker, P.: 1993, Transsentential Meditations. Ups and Downs in Dynamic Semantics, Ph.D. thesis, University of Amsterdam

Dekker, P.: 2002, Something else, in A. N. G. Alberti, K. Balogh (ed.), Proceedings of the Seventh Symposium on Logic and Language, Pecs, 2002

Gawron, J. M.: 1996, Quantification, quantificational domains and dynamic logic, in S. Lappin (ed.), The Handbook of Contemporary Semantic Theory, Basil Blackwell, London

Ginzburg, J.: 1995, Resolving questions, I \& II, Linguistics and Philosophy 18, 459-527, 567-609

Goodman, N.: 1961, About, Mind 70, 1-24

Groenendijk, J.: 1998, Questions in update semantics, in J. Hulstijn and A. Nijholt (eds.), Formal Semantics and Pragmatics of Dialogue, University of Twente

Groenendijk, J.: 1999, The logic of interrogation, in T. Matthews and D. Strolovitch (eds.), The Proceedings of the Ninth Conference on Semantics and Linguistic Theory, CLC Publications

Groenendijk, J. and Stokhof, M.: 1984, Studies on the Semantics of Questions and the Pragmatics of Answers, Ph.D. thesis, University of Amsterdam

Hamblin, C. L.: 1973, Questions in Montague English, Foundation of Language $10,41-53$

Hulstijn, J.: 1997, Structured information states. Raising and resolving issues, in A. Benz and G. Jäger (eds.), Proceedings of MunDial97, University of Munich

Jäger, G.: 1996, Only updates, in P. Dekker and M. Stokhof (eds.), Proceedings of the Tenth Amsterdam Colloquium, ILLC, University of Amsterdam, Amsterdam

Karttunen, L.: 1977, Syntax and semantics of questions, Linguistics and Philosophy 1, 3-44

Krifka, M.: 1992, A compositional semantics for multiple focus constructions, in J. Jacobs (ed.), Informationsstruktur und Grammatik, Linguistische Berichte, Sonderheft 4

Krifka, M.: 2001, For a structured meaning account of questions and answers, in C. Fery and W. Sternefeld (eds.), Audiatur Vox Sapientia. A Festschrift for Arnim von Stechow, pp 287-319, Akademie Verlag, Berlin

Lewis, D.: 1988, Relevant implication, Theoria 18, 161-174

Paul, H.: 1880, Prinzipien der Sprachgeschichte, (8th edition, Tübingen, Niemeyer, 1970)

Reinhart, T.: 1981, Pragmatics and linguisțics: An analysis of sentence topics, Philosophica 27(1), 53-94

Roberts, C.: 1996, Information structure in discourse: Towards an integrated formal theory of pragmatics, in J. Yoon and A. Kathol (eds.), OSU Working Papers in Linguistics 49, pp 91-136

Rooth, M.: 1985, Association with Focus, Ph.D. thesis, University of Massachusetts, Amherst

Rooth, M.: 1992, A Theory of Focus Interpretation, Natural Language Semantics $1,75-116$ 
Schwarzschild, R.: 1999, GIVENness, avoid F and other constraints on the placement of focus, Natural Language Semantics 7(2), 141-177

Vallduvi, E.: 1990, The Informational Component, Ph.D. thesis, University of Pennsylvania, Published by Garland Press in 1992

van Rooy, R.: 1997, The context-dependence of questions and answers, in P. Dekker, M. Stokhof, and Y. Venema (eds.), Proceedings of the Eleventh Amsterdam Colloquium, ILLC, University of Amsterdam, Amsterdam

van Rooy, R.: 2002, Comparing questions and answers

von Fintel, K.: 1995, A minimal theory of adverbial quantification, in B. Partee and H. Kamp (eds.), Context Dependence in the Analysis of Linguistic Meaning, IMS Stuttgart Working Papers, pp. 153-193

von Stechow, A.: 1990, Focusing and backgrounding operators, in W. Abraham (ed.), Discourse Particles, No. 6, pp 37-84, John Benjamins, Amsterdam

Zeevat, H.: 1994, Applying an exhaustivity operator in update semantics., in H. Kamp (ed.), Ellipsis, Tense and Questions, ILLC, Amsterdam, Dyana-2 deliverable R2.2.B 